principe que la "richesse d'une nation est fonction de la "richesse de l'ensemble des pays qui composent celle " nation ".

"Pour tous ces molifs,

"La Chambre de Commerce de Crenoble, eslimant que l'intervention du Ministère des Beaux-Arts dans la question d'ulilisalion des forces hydrauliques ne pourrail que compliquer inutilement, sinon même entraver l'exécution des projets de créalion de chutes d'eau déjà soumis, en l'état, aux Ministères des Travaux publics, de l'Agricullure el des Finances, et donl la solution n'est trop souvent si lente à intervenir, en raison de la multiplicité des formalités administralives dont lear approbation est entourée.

"Emel à l'unanimité de ses membres l'avis que l'intervenlion du Ministre des Beaux-Arts est absolument inutile et qu'elle ne pourrait être que préjudiciable au développement de l'industrie de la houlle blanche, une des richesses nalurelles les plus considérables d'une grande partie de la France ;

"Elle proteste énergiquemenl contre la campagne ayant pour bul ou pour résultal de faire échouer ou d'enrayer les projels nés ou à nathe en vue de l'ulilisation des ressources hydrauliques des Alpes el proteste également contre le classement des sites comme biens nationaux, ce classemenl ne pouvant avoir pour effet que de paralyser l'essor d'indusIries nouvelles, auxquelles le Dauphiné doit, en grande partie, son magnifique développement économique et qui, en donnant anx populalions déshérilées de nos monlagnes la possibilite de vivre désormais sur lear lerriloire, contribuent si puissamment à metlre fin au paupérisme et à enrayer l'émigralion.

"Comme conséquence, la Chambre demande à $M$. le Prójet el à MM. les Représentants du Départernent d'intervenir dans ce sens axprès des Adminislralions consultées pour hâler les formalilés présentes en ce qui concerne les projels en voie d'approbalion."

La Chambre de Commerce de Grenoble a approuvé les termes et les conclusions de ce rapport.

\section{LE NITRURE D'ALUMINIUM}

\section{Les Brevets français relatifs à cette fabrication}

Quels sont les brevets frangais qui sont, ou vonl être, appliqués dans la fameuse industric naissante du nitrure d'aluminium, el quelle est la valeur réelle de ces brevels ? Celle question nous a ćté souvent posée, et pour l'ćludier, sinon pour la résoudre, nous avons abordé l'ćlude de la hrès voluminense liltérature que forme déjà l'ensemble des brevels français publićs jusqüa ce jour rolativement à la fabricalion du nitrure d'aluminium. Nous latisserons de côlé, pour le momenl du moins, les brevels relatifs a la decomposilion de ce nitrurc.

Non seulement celle litlérature très spéciale esl volumineuse : mais elle est terriblement indigeste I Le slyle de presque tous ces brevels n'a que de loinlains rapports avec le bon français ordinaire. Il semble que tous les inventeurs, même français, commencent par penser el rédiger on allemand, pour traduire ensuite et mot à mot en francais. D'autre part, un certain nombre de brevets semblent avoir ćté déposés en vue de gêner ou dérouter les concurrents, et lícrivent avec un grand luxe de détails oiseux des disposilifs imaginés de toute pièce a priori et sans aucune inten. tion de les réaliser jamais. Enfin, et surtout, de mème que, lorsqu'on visite une usine, si on n'èst pas tout à fait spccialiste, on n'aperçoil pas les détails les plus nouveaux et les plus importants, de même, si on n'est pas de la partie, on ne distingue pas facilement dans les brevets relatifs ì la fabricalion des nitrures, les particularités réellement importantes. Or, qui donc peul se dire vraiment " de la partie $n$, en dehors des deux très petits groupes de techniciens rivaux ef concurrents qui poursuivent actuellement, en grand secret, dans deux vallées voisines de la Savoie, la mise a point de la nouvelle fabrication ?

Nous n'avons donc d'autre prétention, dans l'exposé quil va suivre, que de donner un aperç de la complexité du problème. Assurément la question ne restera pas toujours aussi nuageuse ; bientòl poul-être, c'est-à-dire dans un an ou deux, on saura quels sont les brevels réellement utilisés ou ulilisables.

On peut compter unc trentaine de brevets français publiés jusqu'à ce jour pour la fabrication du nitrure d'aluminium. Fn outre, une douzaine de brevets français ont été déposés, mais ne sont pas encore publiés, et, naturellement, ce sont les plus importanls ! Dès lors l'examen des brevets déjà publiés ne peul donner une idée exacte de la. nouvelle industrie, actuellement en pleine évolution.

Nous préscnterons donc simplement une liste des principales particularilés brevetecs jusqu'à ce jour,. $1^{\mathrm{or}}$ juillet 1913. Les letures majuscules désigneront les particularités. qui nous paraissent lombées dans le domaine public en France ; les lettres minuscules désigneront les particularilés qui nous paraissenl au contraire garantics par des brevels particuliers achuellement encore en vigueur.

A. - Ciest en r862 que le niliure d'aluminium a élé découverl, el ses propriélés chimiques essentielles ont été décrites on 1876 , mais il s'agissait de recherehes de laboraloire. La fabrication industrielle de ce nouveau corps est une première parlicularilé imporlante qui a été brevetée par Willson (brevet anglais 2 I 755 du ${ }^{5} 5$ novembre 1895 ). Le brevel français correspondant n'ayant pas été demandé, celte particularité est dans le domaine public en France depuis lc 16 novembre 1896 .

B. - Fabrication industriclle par chauffage, en présence l'azote, d'un mélange d'alumine el de charbon. Brevel Willson du $x 5$ nov. 1895 . Briegleb et Geuther, en 1862 , el Mallel, en 1876 , avaient oblemu le nilrure d'aluminium en partant de l'aluminium mélal. Meme observation que pour $A$.

C. - T.e chaulfige est effectué "dans un four électrique approprié $"$. Brevet anglais de Willson de $18_{95} 5$. Même observation que pour $A$.

D. - Ce four électrique est à arc el à résislance, la résistance étant conslituée par la matière à traiter. Ie lexle du brevet Willson, de 1895 , dit : « Dans un four électrique approprié, préférablement dans un four à arc n, et les trois figures $i, 2$ et 3 du brevel représentent bien, en effet, une électode mobile verlicale, placée à une certaine dislance des matières à traiter, donc engendrant un arc entre elle-même et ces malières. Mais ces mêmcs figures montrent que s'il se prodüit un are entre l'électrode mobile et les malières à traiter, par contre au-delà de cet are et pour gagner la sole conductrice du four, le courant tra- 
verso une cerlaine épaisseur des malières à trailer qui sont bone, en délinilive, chauffées à la fois par l'are qui les surmonle el par le courant qui les traverse, c'est-à-dire à la fois par are et par résistance.

Io brevel américain de Chalmot, du 23 janvicr 1896 , présenle la même particularité du chaulfage mixte par arc et nu résistaneo de la malière à traiter. Ia figure d de la planche I représente bien deux électrodes horizontales cnlièremont noyées dans la matière à liaiter, et à cel égard il semble quón doive considérer le chaulfage comme produit rachusiremont par résistance de la matière à traiter. Mais, d'anle part, l'écartement des deux électrodes est si faible yu'il esl vraisemblable que, dans l'espril de l'auteur, il devait, dans son four, so former un arc; et effectivement le wale dit que les ćlectrodes doivent pouvoir avancer ou reculer "afin de faire varier la longueur de l'arc ou la quantiló de matiòre interposée ontre les deux électrodes et chauffó par le courant électrique allant d'une électrode à l'autro.")

F. - Comme matière premièré devant apporter l'alumihium of en remplaccment de l'alumine, on fait usage des "scls d'aluminium " ou des "composés oxygénés réfraclaires de l'aluminium ". Brevet Willson de 1895 .

li. - Comme malière première devant apporter le carbone, on fail usage de "coke ou de houille " ou de "malieres charbonneuses ". Brevets Willson et de Chalmot.

C. - Comme source d'azote, on emploie le gaz de gazogane. le brevet Willson décrit et figure d'une façon tròs préeise celle particularité ; il en est de même du brevet annéricain de Chalmot, qui est très explicite à cet égard.

II: - On ajoute au mélange un excès de charbon afin de le rendre plus accessible dans loutes ses parties au courant ('azolc ou de gaz azoté qui le traverse. Celte parlicularilé est indiquée très explicitement el motivée avec grands détails dans le brevet de Chalmot dont le correspondant français n'a jamais été demandé. Cette parlicularité est donc dans le domaine public en France depuis le 24 janvier 1897 .

1. - Le jour électrique est constitué par une sole fixe et une électrode verticale mobile disposée au-dessus. Ciest le dispositif décrit el figuré dans le brevet Willson, el aussi figure 6 du brevet de Chalmot.

T. - L'électrode est creuse et sert à l'introduction de l'azote dans le four. Celte parlicularité est représcntée dans 1. brevet Willson figure 4 , et dans le brevel de Chalmol figure 6 , plus haul indiqués.

K. - Le four bedrique cst constitué par une cuve cylindrique, avec deux élcetrodes horizonlales diamélralement opposées, entre lesquelles descendent les malières qui remplissent le four. C'est le four principal (fig. r) du brevet do Chalmot.

I. - Comme gaz azolé, on emploie simplement de l'air atmosphérique. Celte particularité se tromve dans le brevet allcmand de Mehner $\mathrm{N}^{\circ} 88999$, du $\mathrm{r}^{5}$ juin 1895 , dont le correspondanl français est le $\mathrm{N}^{\circ} 25429^{3}$, du 26 février $x 8_{9} 6$. Colle particularité est done dans le domaine public en France depuis l'époque où les annuilés dudit brevet $N^{\circ} 254293$ n'onl plus été payées, et au plus lard depuis le 27 février de l'année rgir.

a. - On emploie comme malière première le carbure d'aluminium fabriqué au préalable; ce carbure, chauffé dans un four électrique en présence d'azote, donne du nitrure d'aluminium. Brevet français Serpek 367 124 du 13 juin rgo6. b. - On emploie comme matière première un mélange de carbure d'aluminium, d'alumine et de charbon. Brevet Sorpek 367 r 34 du 13 juin 1906 .

c. - On ajoule différents ingrédients dans le but d'abaisser la lempéralure de réaction. Différents brevets Serpek :

$I^{\circ}$ On ajoule du cuivre, du fer, de l'acile chlorlydrique, the l'acide sulfureux ( 367 r 34 du 13 juin $x_{906}$ );

$2^{\circ}$ On ajoule du soufre, soit seul, soit combiné (426868 (4) 3 mai rgro).

$3^{\circ}$ On ajoule de l'acide borique ( $\$ 37$ I68 du ro févr. x९r 1 ).

$4^{\circ}$ On ajoule de l'hydrogène au gaz azoté (148 92.4 du 9 décombre Igr $\mathrm{r}$ ).

$5^{\circ}$ On ajoute de l'hydrogène an gaz azolé, et des "oxydes, lydroxydes, carbonates mélalliques ou mélaux n à l'alumine

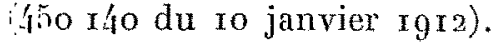

Comme additions ayant pour bul d'abaisser la tempérafure de réaction, on doit citer aussi celles énumérées dans lo Lrevet français 418425 du 24 juin 19 o de la Badische Anilin \& Soda Fabrik : la silice, les oxydes de tilane, de zirconium, de molybdène, du vanadium, du glucinium, du cé1:t:m, de l'uranium, du chrome, ete.

d. - Four bectrique à induction : la malière chauffanle est de la fonte de fer en fusion (brevel Serpek 4 i5 2 i 6 du $\because 6$ juillet $\mathrm{rgog})$.

e. - Chauffage séparé de la bauxile d'une part el du charbon d'autre part, puis réunion des deux et insufflation d'azole. Brevel Serpek 4 I 947 du 26 janvier I910. Mais aucun appareil n'a élé indiqué pour rénliser ce procédé.

f. - Four électrique lournant, à résistance, la résislance élant constituée par des barres en charbon aggloméré disposćes de différentes manières qui ont été indiquées dans los huit brevets Serpck (ou additions) do la liste ci-après :

427066 du 18 mai rgro;

ı $3958 \mathrm{du}$ r $^{\text {er }}$ juin roro (addilion) ;

430553 du 1 I août rgro;

I/4 338 du $3_{\mathrm{I}}$ aoul rg ro (addition) ; r/4 589 du 28 oclobre rgro (addition);

I4 830 du 8 décombro igro (addition);

$4443 \times 6$ du 4 août rgrr;

I5 923 du 4 août rgr (addition).

Ce four électrique est assurément d'une grande originalité, el semble constiluer la caractéristique principale du procédé Serpek.

g. - Four à cuve dans lequel des agglomérés de bauxile et charbon sont chaulfés par lo gaz azoló, lui-même chauffé dans un four électrique accolé an four à cuve ou peu dislant de lui. Brevet Serpek 430822 du 23 août Igro.

h. - Revêtement très réfraclairo pour fours électriques constitué par du nitrure d'aluminiam moulé en briquettes an moyen d'un agglulinant appsopric. Brevets de la Soc. gén. des Nilrures : 436596 du 26 janvier r 9 I ct 438998 du 28 mars Igr.

i. - Four fixe à résislance constituée par une clectrode verticale fixe de section très réduile par rapport aux électrodes d'entrée et de sortie c'u courant dans le four. Brevet français Soc. gén. des Nitrures 450 I 78 du $\times 2$ janvier rgr.

C'est vraisemblablement dans co four qu'on a obtenu, avec de l'alumine pure comme malière première, les très jolis échantillons de nitrure d'aluminium cristallisé qui ont élé répandus depuis un an environ dans différents milieux financièrs et industricls.

j. - Ensemble complexe et ingénieus de lubes toumanls, trémies, tour à oxyder le silicium, etc., en combinaison avec 
le four tournant de la particularité (f) ci-dessus, ayant pour but de réaliser successivement, et sans laisser refroidir les matières alumincuses, ces quatre opérations principales :

$x^{\circ}$ Déshydratation de la bauxite ;

$2^{\circ}$ Addition à la bauxite du coke nécessaire pour permoltre à la réaction de s'effectuer;

$3^{\circ}$ Eliminalion du silicium par vaporisation (et oxydation de ce silicium dans une annexe disposée à cet effet) ;

$4^{\circ}$ Fixation de l'azole, c'est-ìndire transformation de l'alumine en azolure d'aluminium.

Brevet Serpek 427 rog du ig mai rgro.

k. - On chauffe à la lempérature de $1800^{\circ}$ à $1850^{\circ}$ C. qui serail la plus favorable. Brevet Serpek $4 \mathrm{x} \times 03 \mathrm{r}$ du $\mathrm{r}^{\text {er }}$ avril rgog. Mais ce brevet ne démontre pas qu'une température plus álevée, de $1850^{\circ}$ à I $900^{\circ}$ par exemple, ne serait pas encore plus favorable ; et, d'autre part, le brevet n'indique aucun disposilif pour réaliser la régularisation de la température entre $\mathrm{I} 800^{\circ}$ et $\mathrm{I} 850^{\circ} \mathrm{C}$.

1. - On emploie le carborundum comme support de l'alumine qu'on veut transformer en nitrure par chauffage électrique en présence de carbone et d'azote. Brevet Coutagne $4367^{\text {Io }}$ du 3 o janvier r 9 тг.

m. - Four fixe à résistance indépendanle de la matière à trailer. Celte rćsistance étant constituée par des barreaux prismatiques en graphite disposés les uns au-dessus des autres dans une sorte de cheminée verticale, la matière à traiter, mélange pulvérulent d'alumine et de errbone, est versée en haul et tombe en cascade jusqu'au bàs, tandis que le gaz azolé circule en sens inverse de bas en haut. Brevet Coulagne $4367_{8} 8$ du $\mathrm{I}^{\text {er }}$ février $19 \mathrm{rr}$; addition $45 \mathrm{u} 5 \mathrm{I} \mathrm{du}$ 25 février 191 I el addition $156 \mathrm{rg}$ du 27 mai Igr.

n. - Four fixe à résistance indépendante de la matière i traiter. Coltc résistance étant constituée par une série recliligne de morccaux de coke arrangés au milieu du mélange te matières alumineuses et do charbon ; c'est l'application à la fabrication du nitrure d'aluminium du dispositif déjà employé pour la fabrication du carborundum. Brevet Coulagne 437504 du so février igr.

o. - La résislance chauffante esi constituée par une masse de morceaux de coke, entre lesquels le mélange pulvérulent de matières alumineuses et charbonneuses est logé par le simple mélange sans arrangemenl dos morceaux de cokc avec le dit mélange. Brevet Coutagne i 5774 du 29 juin igr I (addition).

). - On réduit l'alumine, en présence d'azote. par les vapeurs de sodium naissant, dans un four tubulaire chauffé par la combustion de gaz de gazogène. Brevet Giulini 45 r 405 du 15 novembre 1912 . Ce procédé ne nous paraft pas devoir être bien avantageux : la dépense de carbonate de soude, de combuslible et de main-d'œurre, et les frais d'entretien d'un four très compliqué, nous semblent des conditions d'infériorité manifestes vis-à-vis des procédés qui réalisent, dans les fours électriques, la réduction de l'alumine par le charbon seul, sans addition de réactifs coûteux. D'ailleurs Serpek ayant breveté l'addition des " oxydes, hydroxydes, carbonates métalliques on métaux " $\left(\mathrm{n}^{\circ} 450\right.$ r 40 du ro janvier rgra), n'objectera-t-il pas que l'addition de carbonate de soude rentre dans les termes de son brevet? Gílini répondra sans doute alors que son carbonate de soude n'est pas un réactif catalytique, à dose homéopathique, et qu'il en ajoute des doses massives... Mais toutes ces discussions ne se produiront vraisemblablement pas si, comme il esi probable, le procédé Giulini ne permet pas de fabriquer économiquement le nitrure.
Comme conclusion, nous nous bornerons à répéter que les brevets ou autres documents techniques publiés jusquil ce jour donnent une idée encore bien vague, et en tout cas très incomplète, de ce que sera la nouvelle industrie du ni. trure d'aluminium. Aussi bien pour le procédé Serpek qué pour le procédé Coulagne, les dispositifs des fours élec. trique actuellement à l'essai, ou en voie d'installation in. dustrielle, sont jalousement tenus secrets. Il faut donc, de toute nécessité, attendre de nouvelles publications, brevels ou mémoires techniques, avant de pouvoir apprécier Ia valeur relative des deux procédés concurrents, et l'impor: tance économique que le meilleur des deux acquerra sans doute, d'une part dans l'industrie de l'aluminium, et d'aulre part dans l'industrie de la synthèse des sels ammoniacaux ou autres produits chimiques azotés.

E.-F. CôtTe.

\section{TRAVAUX PUBLICS}

\section{Congrès National de Navigation Intérieure}

( $4^{\text {me }}$ Session) Nantes, du 16 au 18 juin 1913

Nous avons toujours tenu nos lecteurs au courant des projets proposés pour l'amélioration des voies fluviales françaises. Cette étude ne saurait laisser indifférent toux ceux qui cherchent à développer l'utilisation de nos forces hydrauliques, car la solution de ces deux problèmes est souvent commune. Pour rendre aisément navigables les rivières à forte pente, le Rhône en particulier, on sera amené a construire une série d'écluses, constituant sur la rivière des chutes d'eau d'une très grande puissance, et il serait difficile d'envisager les dépenses considérables engagées dans ces grands travaux, sans compter sur le revenu des Usines hydroélectriques créées pour utiliser ces chutes d'ean.

L'amélioration de la navigation intérieure provoquera ainsi le développement des grandes forces hydrauliques, et l'énergie électrique résultante augmentera l'activité industrielle de la région et, par suite, le tonnage des marchandises dont les mariniers seront les premiers à profiter.

Nous avons signalé, dans un numéro précédent, le Congrès national de navigation intérieure, qui s'est réuni à Nantes; du $x_{6}$ au $\mathrm{r} 8$ juin $\mathrm{rg}_{9} 3$. Nous ne pourons, dans le cadre de cette Revue, reproduire tous les rapports intéressants qui y ont été communiqués ; nous désirons, toutefois, donner quelques extraits de ceux qui sont susceptibles d'intéresser plus particulièrement nos lecteurs.

Nous citerons tout d'abord le remarquable rapport de M. Lavaud, directeur de la Compagnie de Touage et de Remorquage de la Basse-Seine, sur le programme des grands travaux étudiés par le Gouvernement.

\section{PROGRAMME DES GRANDS TRAVAUX DU GOUVERNEMENT}

Constof́ratrons générales. - En dehors de quelqútes avantages propres, la Navigation est en état d'infériorité manifeste par rapport au Chemin de fer. Elle doit dono offrir à ceux qui sont ses clients, ou peuvent le devenir, des différences de prix sensibles. C'est ce qui est non seulement admis, mais proclamé lorsqu'on pose en principe que les tarifs de chemin de fer les plus bas doivent être d'au moins $20 \%$ supérieurs à ceux de la Navigation. Aussi lorsqu'une nouvelle voie navigable, artificielle ou rivière transforméé, est à l'étude, faut-il se pénétrer de cette idée qu'on ne pourra y appliquer que des péages fort réduits. S'ils devaient être 